PROCEEDINGS OF THE

AMERICAN MATHEMATICAL SOCIETY

Volume 128, Number 4, Pages 1117-1124

S 0002-9939(99)05091-1

Article electronically published on August 5, 1999

\title{
POROSITY OF ILL-POSED PROBLEMS
}

\author{
ROBERT DEVILLE AND JULIAN P. REVALSKI
}

(Communicated by Dale Alspach)

\begin{abstract}
We prove that in several classes of optimization problems, including the setting of smooth variational principles, the complement of the set of well-posed problems is $\sigma$-porous.
\end{abstract}

\section{INTRODUCTION AND PRELIMINARIES}

Let $X$ be a completely regular topological space and $f: X \rightarrow \mathbf{R} \cup\{+\infty\}$ be an extended real-valued function which is bounded from below and proper. As usual the term proper means that the domain of $f$, which is the set $\operatorname{dom} f:=\{x \in$ $X: f(x)<+\infty\}$, is non-empty. The problem to minimize $f$ on $X$, i.e. to find $x_{0} \in X$ such that $f\left(x_{0}\right)=\inf \{f(x): x \in X\}=: \inf (X, f)$, will be designated in the sequel by the pair $(X, f)$. The problem $(X, f)$ is called Tykhonov well-posed ([Ty, DoZo $]$ ) if it has a unique solution $x_{0}$ towards which converges every minimizing sequence for $(X, f)$. Recall that a sequence $\left\{x_{n}\right\}_{n=1}^{\infty} \subset X$ is minimizing for $(X, f)$ if $f\left(x_{n}\right) \rightarrow \inf (X, f)$.

Since we do not intend to involve other types of well-posedness (which exist in different settings; see the comprehensive monograph DoZo), from now on, we shall simply say that a problem " $(X, f)$ is well-posed" instead of saying that " $(X, f)$ is Tykhonov well-posed". Let us mention also that the notion " $(X, f)$ is well-posed" is termed in Banach space theory as "the function $f$ has a strong minimum in $X$ " (cf. Ph, DGZ2]). Below often an extended real-valued function $f: X \rightarrow \mathbf{R} \cup\{+\infty\}$ will be supposed to be lower semicontinuous. By this as usual we mean that its epigraph epi $f:=\{(x, t) \in X \times \mathbf{R}: f(x) \leq t\}$ is a closed subset of $X \times \mathbf{R}$ considered with the product topology.

Now let $(Y, d)$ be a complete metric space of bounded from below real-valued functions defined in $X$. The question that has been of significant interest in the last 25 years, for different choices of $Y$, is the following one: given a function $f: X \rightarrow \mathbf{R} \cup\{+\infty\}$ which is bounded from below, proper and lower semicontinuous, what is the structure of the set $T:=\{g \in Y:$ the problem $(X, f+g)$ is well-posed $\}$ ?

Received by the editors March 24, 1998 and, in revised form, June 1, 1998.

1991 Mathematics Subject Classification. Primary 46B20, 49J45.

Key words and phrases. Variational principles, well-posed optimization problems, ill-posed problems, porous sets, porosity.

This paper was initiated during a short visit of the second named author in November 1997, in the University of Bordeaux.

The second author was partially supported by the Bulgarian National Fund for Scientific Research under contract No. MM-701/97. 
The first problem is to prove that the set $T$ is non-empty, i.e. that there exists at least one perturbation $g \in Y$ such that the minimization problem $(X, f+g)$ is well-posed (in particular the perturbed problem has a solution, a fact which might not be true for $f$ itself). A further step is to see how "rich" in $Y$ is the set $T$ of good perturbations. The initial attempts have been to show that $T$ is residual in $Y$, which means that the complement of $T$ in $Y$ is of the first Baire category in $Y$. Equivalently (in our setting), $T$ is residual if it contains a dense and $G_{\delta}$-subset of $Y$. The above line of research led to different variational principles starting with the famous Ekeland variational principle [Ek1, Borwein-Preiss smooth variational principle [BP], Deville-Godefroy-Zizler smooth variational principle [DGZ1], generic well-posedness [LuPa, CK, CKR1, CKR2, and many others, which turned out to have a number of applications in optimization, non-linear analysis, Hamilton-Jacobi equations, geometry of Banach spaces, topology etc.

Our aim is to go further and to show that, under general assumptions, the family of ill-posed problems, i.e. the problems that are not well-posed, forms a $\sigma$-porous set in $Y$.

Definition 1.1. Let $(Z, \rho)$ be a metric space and $A$ be a subset of $Z$. The set $A$ is said to be porous in $Z$ if there exist $\lambda_{0} \in(0,1]$ and $r_{0}>0$ such that for any $x \in Z$ and $r \in\left(0, r_{0}\right]$ there exists $y \in Z$ such that $B\left(y, \lambda_{0} r\right) \subset B(x, r) \cap(Z \backslash A)$. The set $A$ is called $\sigma$-porous in $Z$ if it can be represented as a countable union of porous sets in $Z$.

Here $B(z, t)$ stands for the open ball in $Z$ centered at $z$ and with radius $t>0$. Let us mention that in the above definition the part "for any $x \in Z$ " can be equivalently replaced by "for any $x \in A$ ".

The definition of a porous set given above is stronger than the concept with the same name introduced by Zajíček in metric spaces (cf. e.g. [Za]; the notion from Definition 2.4 is called there "uniformly very porous"). For the following facts one can consult the survey [Za].

Evidently, if $A$ is porous, then so is its closure and any of its subsets. It is easily seen that every porous set in $Z$ is nowhere dense in $Z$. Hence every $\sigma$-porous set is of the first Baire category in $Z$. In the case $Z=\mathbf{R}^{\mathbf{k}}$, every $\sigma$-porous set of $Z$ is also of Lebesgue measure zero. Since it is possible to decompose $\mathbf{R}^{\mathbf{k}}$ into two disjoint subsets, one of which of Lebesgue measure zero and the other one of the first Baire category, the notion of $\sigma$-porosity is a strict refinement of both the notions of measure zero and of the first Baire category. In infinite dimensions, especially in Banach spaces (which is the setting of our main result below), the notion of $\sigma$-porosity is still a strict refinement of the notion of the first Baire category ([Za], Proposition 2.30).

We now list below three situations where the family of ill-posed problems is $\sigma$-porous. These three results are consequences of our main result from the next section. In what follows, given a bounded real-valued function $g$ in a completely regular topological space $X$, by $\|g\|_{\infty}$ we denote its sup-norm $\|g\|_{\infty}=\sup \{|g(x)|$ : $x \in X\}$, and in the case when $X$ is a metric space with metric $\rho$ and $g$ is Lipschitzian, we use $\|g\|_{\text {Lip }}:=\|g\|_{\infty}+\sup \{\mid g(x)-g(y) / \rho(x, y): x, y \in X, x \neq y\}$ for its Lipschitzian norm.

Proposition 1.2. Let $X$ be a complete metric space with a metric $\rho$, and let $f$ : $X \rightarrow \mathbf{R} \cup\{+\infty\}$ be a proper bounded from below lower semicontinuous function. 
Let us define:

$$
Y:=\{g: X \rightarrow \mathbf{R} ; g \text { is bounded and Lipschitzian }\} .
$$

Then the space $Y$, endowed with the norm $\|g\|_{Y}:=\sup \left\{\|g\|_{\infty},\|g\|_{L i p}\right\}$, is a Banach space, and the set $T=\{g \in Y$ : the minimization problem $(X, f+g)$ is well-posed $\}$ has a complement in $Y$ which is $\sigma$-porous in $Y$.

In particular, we could derive from here the weak form of the Ekeland variational principle. Indeed let $\varepsilon>0$. Then there exists $g \in Y,\|g\|_{Y}<\varepsilon$, such that the minimization problem $(X, f+g)$ is well-posed. Let $x_{1} \in X$ be the point where $f+g$ attains its minimum. For every $y \in X$,

$$
f(y)+\varepsilon \rho\left(y, x_{1}\right) \geq f(y)+g(y)-g\left(x_{1}\right) \geq f\left(x_{1}\right)=f\left(x_{1}\right)+\varepsilon \rho\left(x_{1}, x_{1}\right) .
$$

But the existence of $x_{1}$ such that " $f(y)+\varepsilon \rho\left(y, x_{1}\right) \geq f\left(x_{1}\right)$ " is the (weak form of the) Ekeland variational principle (see [Ek2]). Moreover, if $f\left(y_{n}\right)+\varepsilon \rho\left(y_{n}, x_{1}\right) \rightarrow f\left(x_{1}\right)$, the above inequalities show that $f\left(y_{n}\right)+g\left(y_{n}\right) \rightarrow f\left(x_{1}\right)+g\left(x_{1}\right)$, and since $(X, f+g)$ is well-posed, $\left\{y_{n}\right\}_{n=1}^{\infty}$ converges to $x_{1}$. Therefore, the problem $\left(X, f(\cdot)+\varepsilon \rho\left(\cdot, x_{1}\right)\right)$ is well posed (for similar results see [Ge] $)$. Let us notice here that, in general, the set of points $x_{1} \in X$ such that the problem $\left(X, f(\cdot)+\varepsilon \rho\left(\cdot, x_{1}\right)\right)$ is well posed has a complement in $X$ which is not $\sigma$-porous in $X$ (in fact, in general, this set is not residual).

Proposition 1.3. Let $X$ be a completely regular topological space which contains a dense completely metrizable subspace $X_{1}$. This means that there exists a complete metric $\rho$ in $X_{1}$ such that the topology generated by this metric in $X_{1}$ coincides with the topology inherited from $X$. Let $Y:=C(X)$, the Banach space of all bounded and continuous real-valued functions in $X$ equipped with the uniform norm $\|g\|_{\infty}$, and let $f: X \rightarrow \mathbf{R} \cup\{+\infty\}$ be a proper bounded from below lower semicontinuous function which is continuous at the points of its domain. Then the set $T=\{g \in$ $C(X)$ : the minimization problem $(X, f+g)$ is well-posed $\}$ has a complement in $C(X)$ which is $\sigma$-porous in $C(X)$.

In particular, the set $T$ is residual. In the case $f \equiv 0$, the fact that $T$ is residual when $X=X_{1}$ (i.e. the space $X$ is itself a complete metric space) was proved by Lucchetti and Patrone [LuPa, when $X$ is a compact Hausdorff topological space by Čoban and Kenderov [CK] and when $X$ is as in the above proposition by Coban, Kenderov and Revalski in CKR1. In fact, when $f \equiv 0$, the property that $T$ contains a dense and $G_{\delta}$-subset of $C(X)$ characterizes the existence of a dense completely metrizable subspace of $X$ ([CK, [CKR1]). These results are of interest not only in optimization but also in geometry of Banach spaces, because of the fact that the well-posedness of $(X,|g|), g \in C(X), g \neq 0$, is equivalent to the Gâteaux differentiability of the sup-norm $\|\cdot\|_{\infty}$ at $g$, and also in topology in connection with topological games ([KR1, CKR2]). For a survey on other generic properties related to well-posedness, see [KR2].

Proposition 1.4. Let $X$ be a real Banach space and $\left(Y,\|\cdot\|_{Y}\right)$ be a Banach space of bounded continuous functions in $X$ with the following properties:

(i) $\|g\|_{Y} \geq\|g\|_{\infty}$ for any $g \in Y$;

(ii) for any $u \in X$ the function $g_{u}(x):=g(u+x), x \in X$, belongs to $Y$ and $\left\|g_{u}\right\|_{Y}=\|g\|_{Y}$ 
(iii) for every $\alpha>0$ and $g \in Y$ the function $h(x):=g(\alpha x), x \in X$, is again from $Y$;

(iv) there exists a bump function in $Y$, i.e. a bounded continuous function $b: X \rightarrow$ $\mathbf{R}$ such that the support of $b, \operatorname{supp} b:=\{x \in X: b(x) \neq 0\}$, is non-empty and bounded.

Now let $f: X \rightarrow \mathbf{R} \cup\{+\infty\}$ be a proper bounded from below lower semicontinuous function. Then, under the above assumptions, the set $T=\{g \in Y$ : the minimization problem $(X, f+g)$ is well-posed $\}$ has a complement in $Y$ which is $\sigma$-porous in $Y$.

In particular, the set $T$ contains a dense and $G_{\delta}$-subset of $Y$ : this is the DevilleGodefroy-Zizler variational principle (see [DGZ1, DGZ2]). If we apply the above result to the Banach space $Y$ of bounded Lipschitzian and $C^{1}$-smooth functions, endowed with the norm $\|g\|_{Y}:=\sup \left\{\|g\|_{\infty},\|g\|_{\text {Lip }}\right\}$, we obtain:

Corollary 1.5. Let $X$ be a real Banach space such that there exists a Lipschitz and $C^{1}$-smooth function $b: X \rightarrow \mathbf{R}$ which is not identically equal to zero and with bounded support. Let $f: X \rightarrow \mathbf{R} \cup\{+\infty\}$ be a proper bounded from below lower semicontinuous function. Then the set $T=\{g \in Y$ : the minimization problem $(X, f+g)$ is well-posed $\}$ has a complement in $Y$ which is $\sigma$-porous in $Y$.

In particular, the set $T$ contains a dense and $G_{\delta}$-subset of $Y$. As shown in [DGZ2], this gives a number of applications, for example in Hamilton-Jacobi equations.

Let us mention that in all the above results, the complement of $T$ in $Y$ is $\sigma$-porous in $Y$, but is not porous in general.

Let us mention that results on porosity are obtained for Fréchet differentiation of convex functions in $[\mathrm{PZ}$ and for a class of best approximation problems in uniformly convex Banach spaces in [DBMP]. The above three propositions will follow easily from a general theorem which will be stated and proved in the next section.

\section{MAIN RESUlT}

Before proving the main theorem we recall two characterizations of well-posedness which will be needed later.

Let $X$ be a completely regular topological space and $h: X \rightarrow \mathbf{R} \cup\{+\infty\}$ be a proper bounded from below function. For a given $\varepsilon>0$ let us denote by $\operatorname{Lev}_{X, h}(\varepsilon)$ the sublevel set of $h$ of height $\inf (X, h)+\varepsilon$, i.e. the set $\{x \in X: h(x) \leq \inf (X, h)+$ $\varepsilon\}$. Obviously $\operatorname{Lev}_{X, h}(\varepsilon)$ is non-empty for any $\varepsilon>0$. The sets $\operatorname{Lev}_{X, h}(\varepsilon)$ are closed provided $h$ is lower semicontinuous, and have non-empty interior if $h$ is continuous at the points of dom $h$. Finally, if $\varepsilon_{1} \leq \varepsilon_{2}$, then $\operatorname{Lev}_{X, h}\left(\varepsilon_{1}\right) \subset \operatorname{Lev}_{X, h}\left(\varepsilon_{2}\right)$. Observe that $\bigcap\left\{\operatorname{Lev}_{X, h}(\varepsilon): \varepsilon>0\right\}$ is exactly the set (possibly empty) of the minimizers of $h$ in $X$.

The following proposition is taken from [CKR1].

Proposition 2.1 ([CKR1], Proposition 1.5, Corollary 1.6). Let $h: X \rightarrow \mathbf{R} \cup\{+\infty\}$ be a proper, bounded from below and lower semicontinuous function. Then the minimization problem $(X, h)$ is well-posed if and only if it has unique solution $x_{0} \in X$ such that for every open set $U \subset X$ containing $x_{0}$ there exists $\varepsilon>0$ such that $\operatorname{Lev}_{X, h}(\varepsilon) \subset U$. In particular, if $h$ is continuous at each point of $\operatorname{dom} h$, then $(X, h)$ is well-posed if and only if there exists a unique solution $x_{0} \in X$ for which the (countable) family $\left\{\operatorname{Lev}_{X, h}(1 / n)\right\}_{n=1}^{\infty}$ is a local base of neighborhoods. 
The above proposition is a topological counterpart of the following characterization of well-posedness in complete metric spaces due to Furi and Vignoli [FV].

Proposition 2.2 ([FV]). Let $X$ be a complete metric space and $h: X \rightarrow \mathbf{R} \cup$ $\{+\infty\}$ be a proper bounded from below lower semicontinuous function. Then the minimization problem $(X, h)$ is well-posed if and only if

$$
\inf \left\{\operatorname{diam}\left(\operatorname{Lev}_{X, h}(\varepsilon)\right): \varepsilon>0\right\}=0 .
$$

Here the symbol $\operatorname{diam}(A)$ denotes the diameter of the set $A$ in the metric space $X$. Below we shall consider a topological space $X$ which contains a dense completely metrizable subspace $X_{1}$. In such a situation, when $A$ is a subset of $X$ which intersects $X_{1}$, and we write $\operatorname{diam}\left(A \cap X_{1}\right)$, we have in mind the diameter of the set $A \cap X_{1}$ with respect to the metric in $X_{1}$.

The following corollary will be needed later as well.

Corollary 2.3. Let $X$ be a completely regular topological space and $h: X \rightarrow \mathbf{R} \cup$ $\{+\infty\}$ be a proper bounded from below lower semicontinuous function which is continuous at the points of $\operatorname{dom} h$. Let $X$ contain a dense completely metrizable subspace $X_{1}$. Suppose that

$$
\inf \left\{\operatorname{diam}\left(\operatorname{Lev}_{X, h}(\varepsilon) \cap X_{1}\right): \varepsilon>0\right\}=0 .
$$

Then the minimization problem $(X, h)$ is well-posed.

Proof. Observe that by the continuity of $h$ at the points of $\operatorname{dom} h$, the set $\operatorname{Lev}_{X, h}(\varepsilon)$ has non-empty interior for every $\varepsilon>0$. Hence, $\operatorname{Lev}_{X, h}(\varepsilon) \cap X_{1} \neq \emptyset$ for each $\varepsilon>0$. Moreover, again by the fact that $h$ is continuous at the points of $\operatorname{dom} h$ we have $\inf (X, h)=\inf \left(X_{1}, h\right)$. Hence, for every $\varepsilon>0$

$$
\operatorname{Lev}_{X, h}(\varepsilon) \cap X_{1}=\operatorname{Lev}_{X_{1}, h \mid X_{1}}(\varepsilon),
$$

where $h \mid X_{1}$ means the restriction of $h$ to $X_{1}$.

Then, by Proposition 2.2, the minimization problem $\left(X_{1}, h \mid X_{1}\right)$ is well-posed with unique solution $x_{0} \in X_{1}$. Moreover, by Proposition 2.1 the sets

$$
\left\{\operatorname{Lev}_{X_{1}, h \mid X_{1}}(1 / n)\right\}_{n=1}^{\infty}
$$

form a local base for $x_{0}$ in $X_{1}$. Now using (2.1), the density of $X_{1}$ in $X$ and the regularity of $X$, it is a routine matter to check that the sets $\left\{\operatorname{Lev}_{X, h}(1 / n)\right\}_{n=1}^{\infty}$ form a local base for $x_{0}$ in $X$. Therefore, by Proposition 2.1, the problem $(X, h)$ is well-posed.

Now we are ready to formulate and prove our main result. Below, for a function $h: X \rightarrow \mathbf{R}$ and $X_{1} \subset X$, the symbol supp $h \mid X_{1}$ means the support of $h$ in $X_{1}$, i.e. the set $\left\{x \in X_{1}: h(x) \neq 0\right\}$.

Theorem 2.4. Let $X$ be a completely regular topological space which contains a dense completely metrizable subspace $X_{1}$. Suppose that $\left(Y,\|\cdot\|_{Y}\right)$ is a real Banach space of bounded continuous functions in $X$ such that:

(a) $\|g\|_{Y} \geq\|g\|_{\infty}$ for every $g \in Y$;

(b) for every natural number $n$, there exists a positive constant $M_{n}$ such that for any point $x \in X_{1}$ there exists a function $h_{n}: X \rightarrow[0,1]$, such that $h_{n} \in Y$, $\left\|h_{n}\right\|_{Y} \leq M_{n}, h_{n}(x)=1$ and $\operatorname{diam}\left(\operatorname{supp} h \mid X_{1}\right)<1 / n$. 
Let $f: X \rightarrow \mathbf{R} \cup\{+\infty\}$ be a proper bounded from below lower semicontinuous function and suppose that either $X=X_{1}$ (i.e. $X$ itself is a complete metric space), or that $f$ is continuous at the points of $\operatorname{dom} f$. Then the set $T=\{g \in Y$ : the minimization problem $(X, f+g)$ is well-posed $\}$ has a complement in $Y$ which is $\sigma$-porous in $Y$.

Remark 2.5. Let us immediately mention that the results from Propositions 1.2, 1.3 and 1.4 are particular cases from the above scheme. Indeed for Proposition 1.3 when $Y=\left(C(X),\|\cdot\|_{\infty}\right)$, (a) is clear and (b) follows by the fact that $X$ is completely regular (hence $M_{n}=1$ for every $n=1,2, \ldots$ ).

For Proposition 1.2 we have $X=X_{1}$ and again (a) is clear. To verify (b) let the metric in $X$ be $\rho$ and $n$ be a fixed natural number. Then given $x \in X$ we take $h_{n}(y):=\sup \{0,1-2 n \rho(x, y)\}, y \in X$. It is easily seen that $h_{n}$ satisfies (b) above and is Lipschitzian with constant $2 n$. Hence we have just to put $M_{n}=1+2 n$.

As to Proposition 1.4, we are again in the situation $X=X_{1}$. The condition (a) above is exactly (i), while (b) can be easily checked to be a consequence of (ii), (iii) and (iv) from the formulation of this proposition. Indeed, for the latter, let $b$ be the bump function guaranteed by condition (iv) of Proposition 1.4. We may think that $b(0)=1, b$ takes its values in $[0,1]$ and that $\operatorname{supp} b \subset B(0,1)$. Then, given a natural $n$, put $M_{n}:=\left\|b_{n}\right\|_{Y}$, where $b_{n}(y):=b(2 n y), y \in X$. By (iii) $b_{n}$ belongs to $Y$. Now given $x \in X_{1}=X$, just take $h_{n}(\cdot)=b_{n}(\cdot-x)$. The last functions is from $Y$ and has the same norm as $b_{n}$ because of (ii).

Proof of Theorem 2.4. Let $n$ be an arbitrary natural number and consider the set:

$$
T_{n}:=\left\{g \in Y: \inf \left\{\operatorname{diam}\left(\operatorname{Lev}_{X, f+g}(\varepsilon) \cap X_{1}\right): \varepsilon>0\right\}<\frac{1}{n}\right\} .
$$

By the assumptions of the theorem (namely, either $X=X_{1}$ or, if this is not the case, that $f$ (and hence $f+g, g \in Y$ ) is continuous at the points of $\operatorname{dom} f$ ) and by Proposition 2.2 and Corollary 2.3 it is seen that $\bigcap_{n=1}^{\infty} T_{n} \subset T$. Hence, the proof will be completed if we show that each $Y \backslash T_{n}$ is porous in $Y$.

To this end fix some arbitrary natural number $n$ and let

$$
\lambda_{n}:=\frac{1}{2\left(1+2 M_{n}\right)},
$$

where $M_{n}$ is the constant given by assumption (b) of the theorem. Let $r_{n}=1$. We claim that $\lambda_{n}$ and $r_{n}$ satisfy the requirements of Definition 1.1.

Indeed, fix arbitrary $g \in Y$ and $r \in\left(0, r_{n}\right]$. In order to complete the proof, it is sufficient to find $g_{n} \in Y$ such that

$$
B\left(g+g_{n}, \lambda_{n} r\right) \subset B(g, r) \cap T_{n} .
$$

Take $\varepsilon_{n}>0$ and $\varepsilon_{n}<r /\left(2 M_{n}\right)$ and let $x_{n} \in X$ be such that

$$
(f+g)\left(x_{n}\right)<\inf (X, f+g)+\varepsilon_{n} .
$$

Due to the assumptions of the theorem we may assume that $x_{n} \in X_{1}$. Indeed, if $X=X_{1}$, this is clear. If not, then $f$ must be continuous at the points of its domain. Hence $f+g$ is continuous at the points of its domain and consequently $\inf (X, f+g)=\inf \left(X_{1}, f+g\right)$. Hence, $x_{n}$ can be taken from $X_{1}$.

Further, by assumption (b) of the theorem, there exists a function $h_{n}: X \rightarrow$ $[0,1]$, so that $h_{n}\left(x_{n}\right)=1, \operatorname{diam}\left(\operatorname{supp} h_{n} \mid X_{1}\right)<1 / n, h_{n}$ belongs to $Y$ and $\left\|h_{n}\right\|_{Y} \leq$ 
$M_{n}$. We define the function $g_{n} \in Y$, as

$$
g_{n}(x):=-\alpha_{n} h_{n}(x), x \in X, \text { where } \alpha_{n}:=\frac{r\left(1-\lambda_{n}\right)}{M_{n}} .
$$

We claim that (2.3) is satisfied. Indeed, let $h \in B\left(g+g_{n}, \lambda_{n} r\right)$. Then $h=g+g_{n}+h^{\prime}$ for some $h^{\prime} \in Y$ with $\left\|h^{\prime}\right\|_{Y}<\lambda_{n} r$. Hence we have the following chain of inequalities and equalities having in mind (2.5):

$$
\begin{aligned}
\|g-h\|_{Y} & =\left\|g-g-g_{n}-h^{\prime}\right\|_{Y} \leq\left\|g_{n}\right\|_{Y}+\left\|h^{\prime}\right\|_{Y} \\
& <\alpha_{n} M_{n}+\lambda_{n} r=\frac{r\left(1-\lambda_{n}\right)}{M_{n}} M_{n}+\lambda_{n} r=r .
\end{aligned}
$$

This shows that $h \in B(g, r)$. Therefore, it remains to show that such an $h$ belongs also to $T_{n}$. For this it is enough to show that

$$
\operatorname{Lev}_{X, f+h}(\varepsilon) \cap X_{1} \subset \operatorname{supp} h_{n} \mid X_{1} \text { for some } \varepsilon>0 .
$$

Obviously for the last inclusion it is enough to verify that

$$
(f+h)(x)>\inf (X, f+h)+\varepsilon \text { for some } \varepsilon>0 \text { and every } x \in X_{1} \backslash \operatorname{supp} h_{n} \mid X_{1} .
$$

Let $h=g+g_{n}+h^{\prime}$, where $h^{\prime} \in Y$ and $\left\|h^{\prime}\right\|_{Y}<\lambda_{n} r$. In the following chain of equalities and inequalities we will use several times the fact that $\left\|h^{\prime}\right\|_{\infty} \leq\left\|h^{\prime}\right\|_{Y}$. Fix an arbitrary $x \in X_{1} \backslash \operatorname{supp} h_{n} \mid X_{1}$. Having in mind also the choice of $h_{n}, g_{n}$ (see (2.5)) and $x_{n}$ (see (2.4)) we obtain:

$$
\begin{aligned}
(f+h)(x) & =\left(f+g+g_{n}+h^{\prime}\right)(x)=\left(f+g+h^{\prime}\right)(x) \\
& \geq(f+g)(x)-\lambda_{n} r \geq \inf (X, f+g)-\lambda_{n} r \\
& \geq(f+g)\left(x_{n}\right)-\varepsilon_{n}-\lambda_{n} r \\
& =\left(f+g+g_{n}\right)\left(x_{n}\right)+\alpha_{n}-\varepsilon_{n}-\lambda_{n} r \\
& \geq\left(f+g+g_{n}+h^{\prime}\right)\left(x_{n}\right)+\alpha_{n}-\varepsilon_{n}-2 \lambda_{n} r \\
& \geq \inf \left(X, f+g+g_{n}+h^{\prime}\right)+\alpha_{n}-\varepsilon_{n}-2 \lambda_{n} r \\
& =\inf (X, f+h)+\alpha_{n}-\varepsilon_{n}-2 \lambda_{n} r .
\end{aligned}
$$

Hence (2.7) will be proved if we show that $\alpha_{n}-\varepsilon_{n}-2 \lambda_{n} r>0$. By the choice of $\alpha_{n}$ in $(2.5)$ and the fact that $\varepsilon_{n}<r /\left(2 M_{n}\right)$, we have :

$$
\alpha_{n}-\varepsilon_{n}-2 \lambda_{n} r>r\left(\frac{1-\lambda_{n}}{M_{n}}-\frac{1}{2 M_{n}}-2 \lambda_{n}\right) .
$$

By the choice of $\lambda_{n}$ in (2.2), the expression in the brackets above is zero. This completes the proof of the porosity of $T_{n}$.

Remark 2.6. It is seen from Proposition 2.2 and the proof of the above theorem, that when $X=X_{1}$ (i.e. when $X$ is itself a complete metric space) the set of wellposed perturbations $T$ is exactly the intersection of all $T_{n}$. Using condition (a) of Theorem 2.4, it can be seen that each $T_{n}$ is open in $Y$. Therefore, it follows that, when $X=X_{1}$, the set $T$ is a $G_{\delta}$-subset of $Y$ whose complement $Y \backslash T$ is $\sigma$-porous in $Y$.

Remark 2.7. A close look at the proof that each $Y \backslash T_{n}$ is porous shows that we never used that $r_{n}$ was chosen to be equal to 1 . The result is true for an arbitrary $r_{n}>0$. In this way we have shown that the set $Y \backslash T_{n}$ has a property stronger than the notion of porosity from Definition 1.1. This stronger property is called "global very porosity" in [Za]. 


\section{REFERENCES}

[BP] J. Borwein and D. Preiss, A smooth variational principle with applications to subdifferentiability and to differentiability of convex functions, Trans. Am. Math. Soc. 303(1987), 517-527. MR 88k:49013

[CK] M.M. Čoban and P.S. Kenderov, Dense Gâteaux differentiability of the sup-norm in $C(T)$ and the topological properties of T, Compt. rend. Acad. bulg. Sci. 38(1985), 1603-1604. MR 87h:46070

[CKR1] M.M. Čoban, P.S. Kenderov and J.P. Revalski, Generic well-posedness of optimization problems in topological spaces, Mathematika 36(1989), 301-324. MR 91c:90119

[CKR2] M.M. Čoban, P.S. Kenderov and J.P. Revalski, Topological spaces related to the BanachMazur game and the generic properties of optimization problems, Set-valued Analysis 3(1995), 263-279. MR 96h:90172

[DBMP] F.S. De Blasi, J. Myjak and P. Papini, Porous sets in best approximation theory, J. London Math. Soc. 44(1991), 135-142. MR 92h:41066

[DGZ1] R. Deville, G. Godefroy and V. Zizler, A smooth variational principle with applications to Hamilton-Jacobi equations in infinite dimensions, J. Functional Analysis 111 (1993), 197-212. MR 94b:49010

[DGZ2] R. Deville, G. Godefroy and V. Zizler, Smoothness and renormings in Banach spaces, Pitman monographs and Surveys in Pure and Appl. Math., Longman Scientific \& Technical, 1993. MR 94d:46012

[DoZo] A. Dontchev, T. Zolezzi, Well-posed optimization problems, Lect. Notes in Math. \# 1543, Springer Verlag, Berlin, 1993. MR 95a:49002

[Ek1] I. Ekeland, On the variational principle, J. Math. Anal. Appl., 47(1974), 324-353. MR 49:11344

[Ek2] I. Ekeland, Nonconvex minimization problems, Bull. Amer. Math. Soc., Vol. 1(1979), 443-474. MR 80h:49007

[FV] M. Furi and A. Vignoli, About well-posed minimization problems for functionals in metric spaces, J. Opt. Theory Appl. 5(1970), 225-229. MR 41:9075

[Ge] P.Gr. Georgiev, Strong Ekeland's variational principle, Strong Drop theorem and applications, J. Math. Anal. Appl. 131(1988), 1-21. MR 89c:46019

[KR1] P.S. Kenderov and J.P. Revalski, The Banach-Mazur game and generic existence of solutions to optimization problems, Proc. Amer. Math. Soc. 118(1993), 911-917. MR 93i:49012

[KR2] P.S. Kenderov and J.P. Revalski, Generic well-posedness of optimization problems and the Banach-Mazur game, in Recent developments in well-posed variational problems, (R. Lucchetti and J.P. Revalski, eds), Mathematics and its Applications \# 331, Kluwer Academic Publishers, Dordrecht, 1995, pp. 117-136. MR 96g:49004

[LuPa] R. Lucchetti and F. Patrone, Sulla densità e genericità di alcuni problemi di minimo ben posti, Bollettino U.M.I. 15-B(1978), 225-240. MR 58:13711

[Ph] R.R. Phelps, Convex Functions, Monotone Operators and Differentiability, Lect. Notes in Math. \#1364, Springer Verlag, Berlin, 1989. MR 94f:46055

[PZ] D. Preiss and L. Zajíček, Fréchet differentiation of convex functions in a Banach space with a separable dual, Proc. Amer. Math. Soc. 91(1984), 202-204. MR 87f:46072

[Ty] A.N. Tykhonov, On the stability of the functional optimization problem, USSR J. Comp. Math. Math. Phys. 6(1966), 631-634. MR 33:6467

[Za] L. Zajíček, Porosity and $\sigma$-porosity, Real Anal. Exchange, 13(1987-88), 314-350. MR 89e:26009

Laboratoire de Mathématiques, Université de Bordeaux, 351, Cours de la Libération, 33400 Talence, France

E-mail address: deville@math.u-bordeaux.fr

Institute of Mathematics, Bulgarian Academy of Sciences, Acad. G. Bonchev Street, BLOCK 8, 1113 SOFia, Bulgaria 